\title{
Impact of forested riparian areas on stream morphology using aerial LiDAR
}

\section{METHODS}

With the growing availability of high-resolution classified LiDAR (including freely-available statewide coverage within lowa), it has become possible to address these issues within a GIS environment.

Using the lowa Department of Natural Resource's digitized top of bank data, stream width was extracted on regularly-spaced transects created perpendicular to the stream centerline along the channel in seven lowa HUC 10 watersheds (Fig 2). At the end-points of each transect using first-returr LiDAR, canopy heights were obtained (Fig 3). These variables were analyzed statistically and used to seek correlations between the width residuals and riparian land cover (Fig 4 \& 5)

\section{OBJECTIVES}

1. Investigate relationships between riparian land use and channel stability

2. Assess the role of large woody debris in channe morphology and complexity, and aquatic habitat

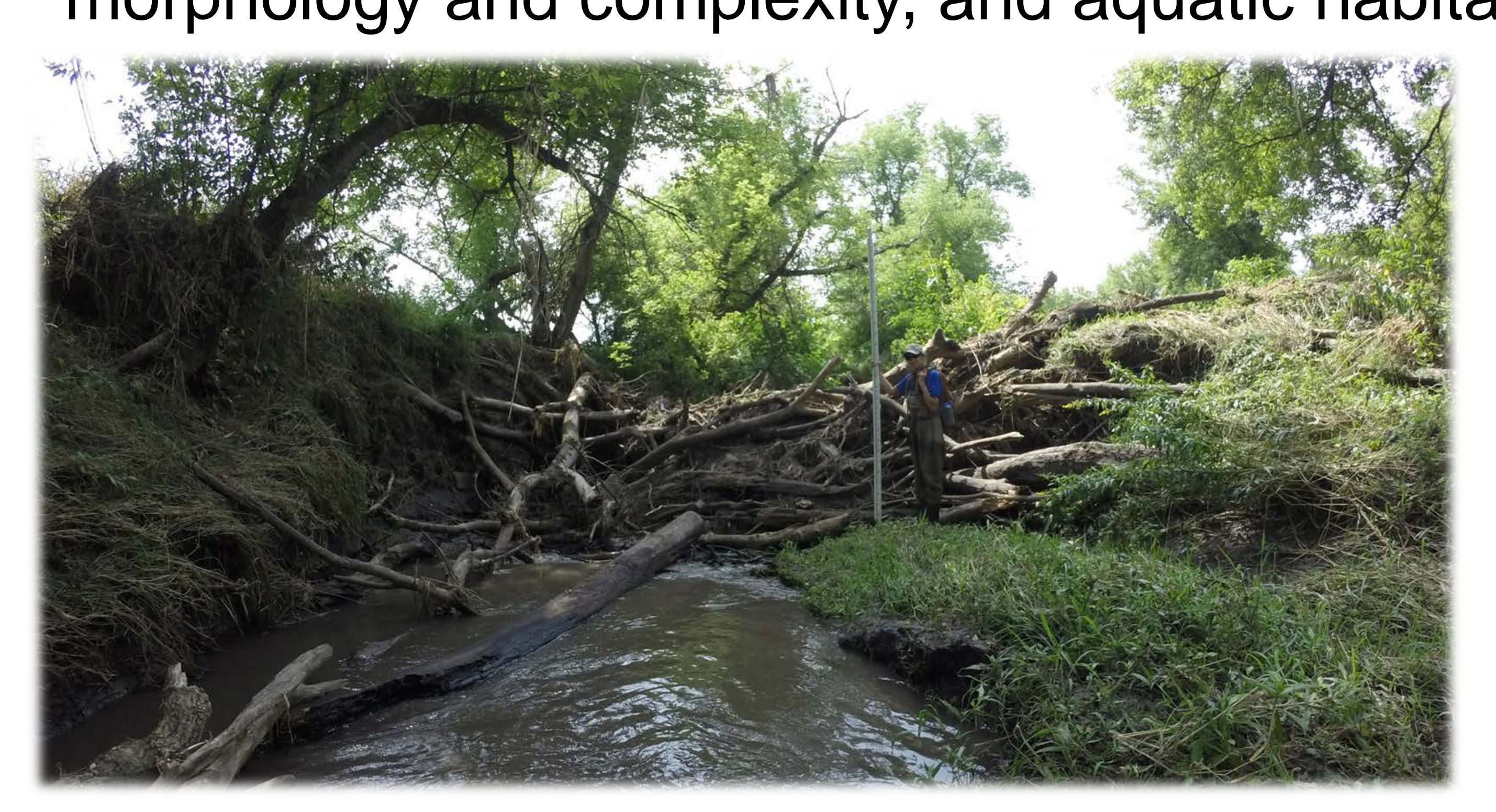

Fig 1. Dam jam of LWD. Photo by W. Beck.

\section{IMPACT}

This research could help to guide individual and institutional practice of riparian land management and stream restoration practice. While managers of waterways and surrounding infrastructure often remove large wood from streams due to perceived risks, this research could provide the observationa basis for re-evaluating this practice in some settings.

\section{INTRODUCTION}

Trees are often planted on stream banks for shade, stabilization, and filtration. Large wood is also introduced within channel structures like engineered jams and habitat for instream species. These practices simulate the geomorphic benefits of natural riparian forest and large wood, but those benefits remain contentious in Midwestern streams. Prior work on the effects of riparian vegetation on stream channel geometry and stability largely have focused on reaches of streams with limited extent, potentially leading to divergent results with little statistical power.

Where channels passing through forested areas have been found to be wider and more unstable, the cause of this relationship has not been well established. Two leading hypotheses are

a) Grass/pasture vegetation promotes sediment accretion on streambanks

b) Streams passing through forest are subject to inputs of large wood, which can increase flow resistance and deflect hydraulic energy toward banks (Fig. 1)

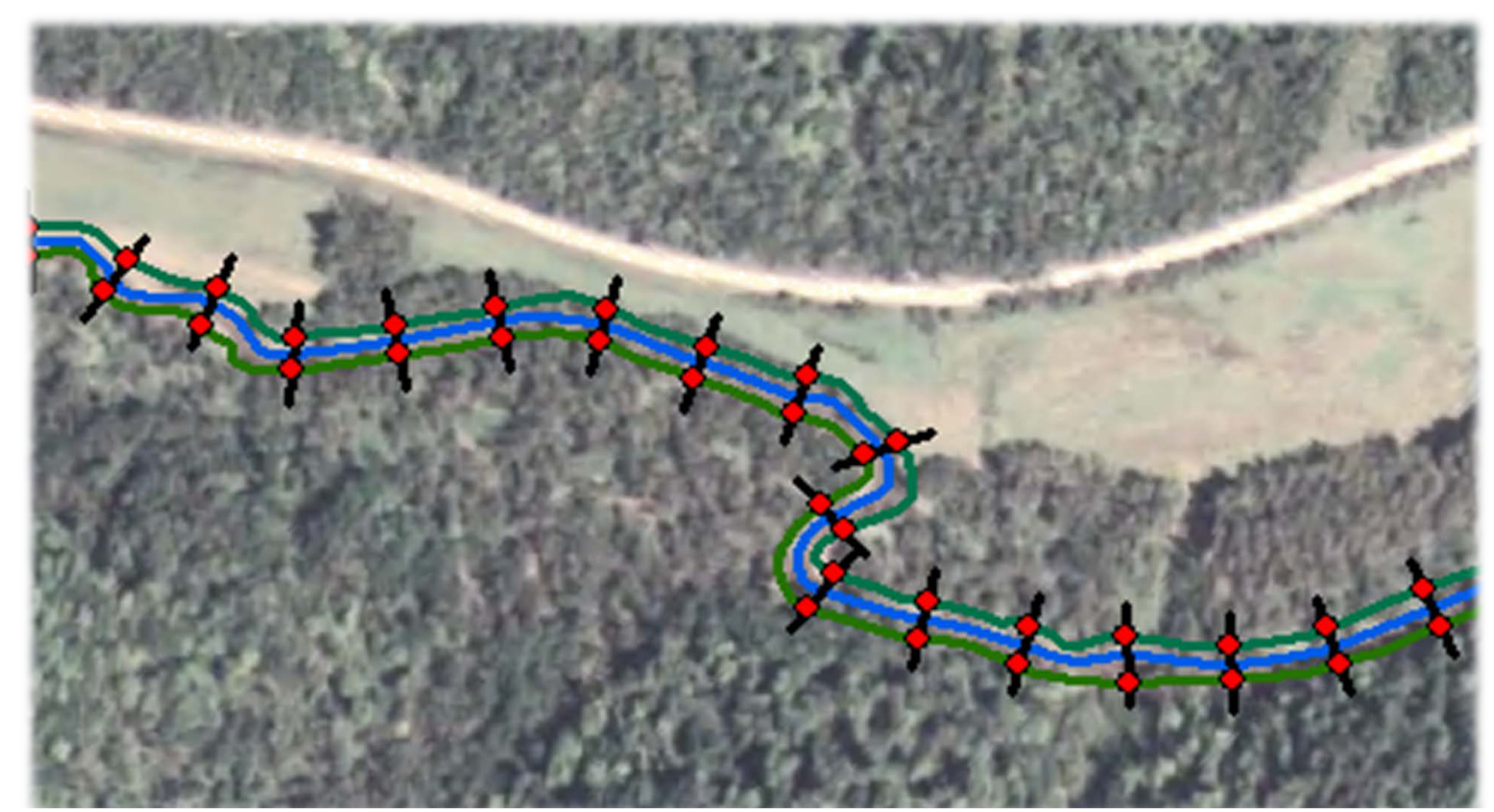

Fig 2. Example of vector data used in the analysis on Paint Creek

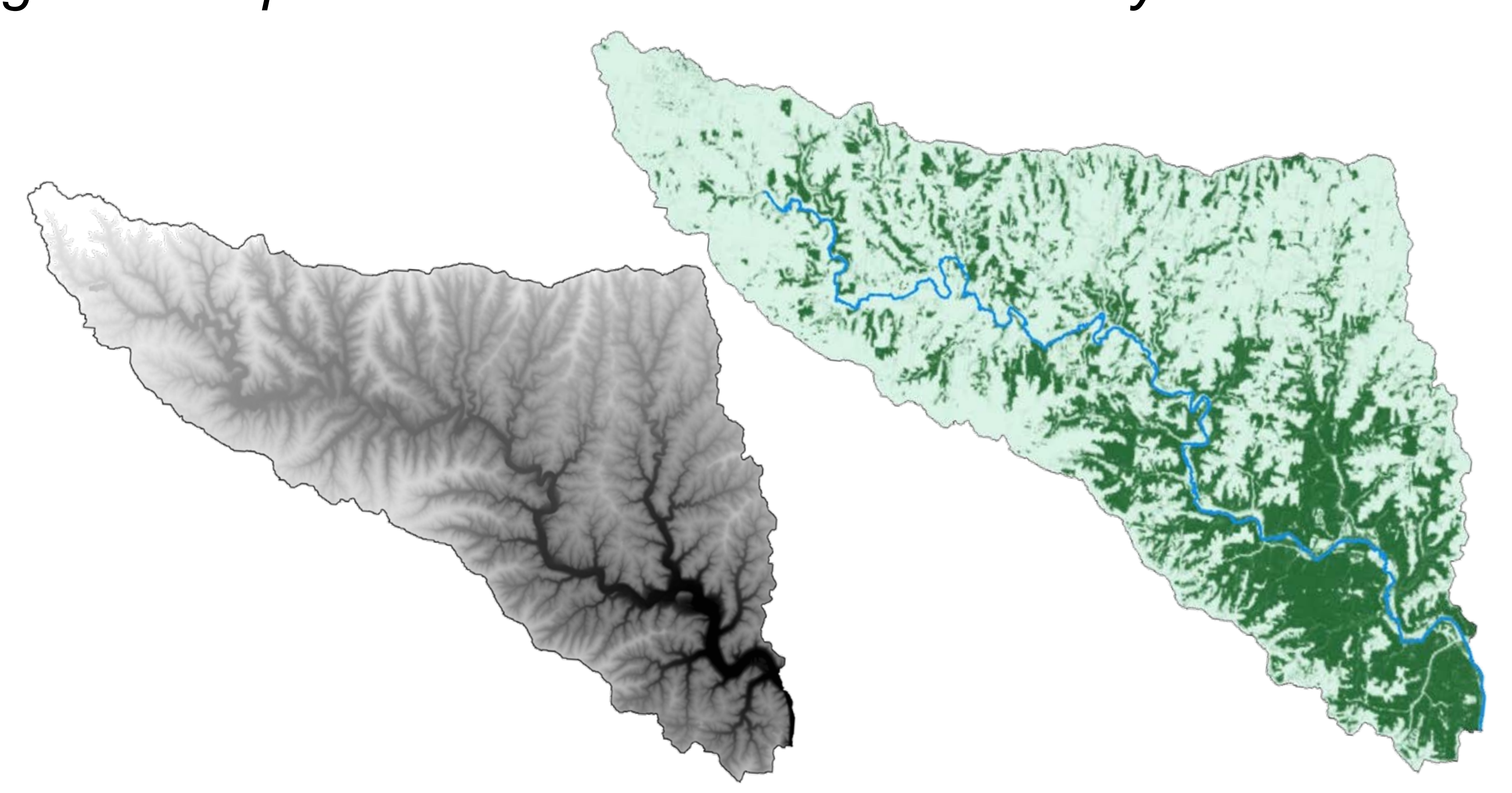

Fig 3. Digital Elevation Model (L) and LiDAR derived canopy cover (R)

\section{PRELIMINARY RESULTS}
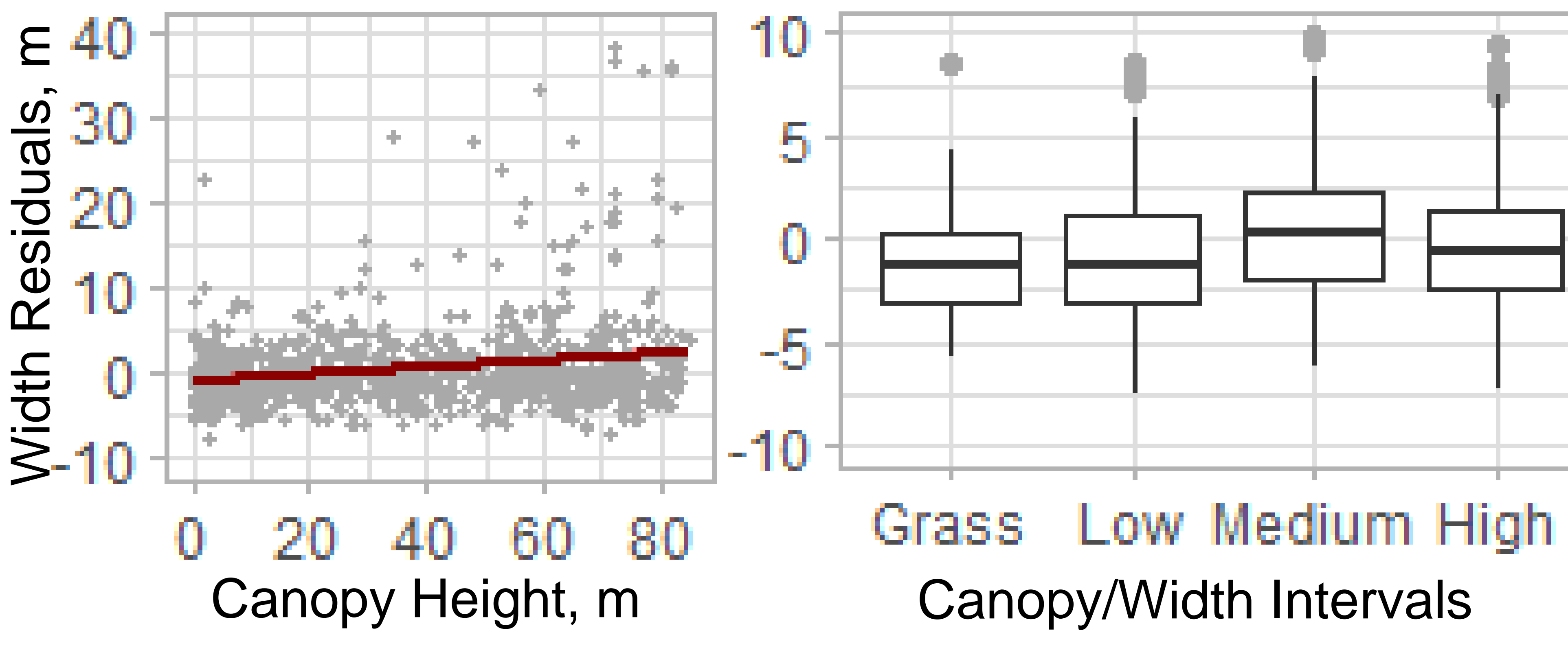

Fig 4. (Left) Scatter plot displaying positive residuals of interest Fig 5.(Right) Boxplots displaying canopy/width split into intervals $<0.5$ (Low), 0.5>\&<1.5 (Medium), >1.5 (High). Any lower values set to Grass.

The expected result of Objective 1 was a partial correlation between land cover and channel width residuals that suggests that, on average, forested channels are indeed wider than unforested. The residuals in Fig 3 also tend to be more positive, variable, and less spatially uniform. A similar analysis conducted on the EPA's Wadeable Streams dataset suggested that this relationship could be at least partly explained by the greater abundance of LWD in forested reaches (Fig 6). Objective 2 will be further investigated in the field at selected sites where physical and biological variables will be collected following the lowa Department of Natural Resource's BioNet protocols.

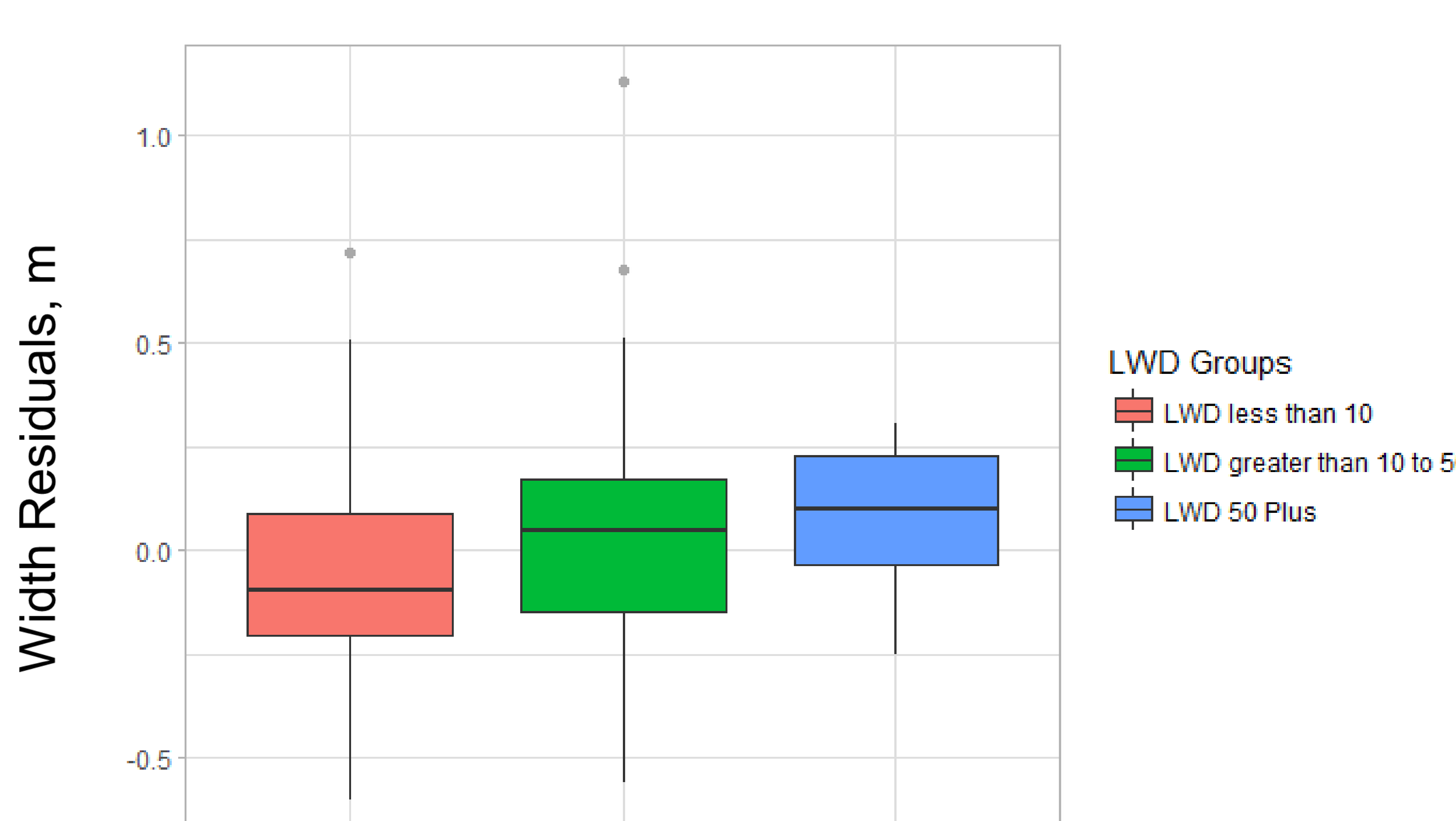

Fig 6. Midwest Wadable Streams data displaying \# of LWD pieces in bankfull channel per reach split into groups. residuals more positive when LWD abundance is greater 